\title{
Caveolin-1 Modulates Docetaxel-Induced Cell Death in Breast Cancer Cell Subtypes through Different Mechanisms
}

Jinho Kang, $\mathrm{BS}^{1}$
Joo Hee Park, MD
Hye Jin Lee, $\mathrm{BS}^{1}$
Ukhyun Jo, PhD'
Jong Kuk Park, PhD²
Jae Hong Seo, MD, PhD'
Yeul Hong Kim, MD, PhD'
Insun Kim, MD, PhD
Kyong Hwa Park, MD, PhD'1

${ }^{1}$ Division of Oncology/Hematology, Department of Internal Medicine,

Korea University College of Medicine, Seoul, ${ }^{2}$ Division of Radiation Cancer Biology, Korea Institute of Radiological and Medical Sciences, Seoul,

${ }^{3}$ Department of Pathology,

Korea University College of Medicine, Seoul, Korea

\begin{abstract}
Purpose
Caveolin-1 (CAV-1) expression is more associated with basal-like cancers than estrogen receptor- or ErbB-2-expressing breast cancers. However, the biological relevance of different levels of CAV-1 expression according to subtype in the epithelial compartment of breast cancer remains unclear.
\end{abstract}

\section{Materials and Methods}

We investigated whether CAV-1 functions as a tumor suppressor and/or modulator of the cytotoxic activity of docetaxel (DTX) in subtypes of breast cancer using in vitro and xenograft models.

\section{Results}

The levels of CAV-1 expression were closely associated with DTX sensitivity in triple-negative breast cancer cells. In addition, CAV-1 significantly inhibited cell proliferation and modulated DTX-induced apoptosis through cell cycle arrest in the G2/M phase. The mechanisms underlying DTX-induced apoptosis differed in breast cancers according to the levels of CAV1 expression. DTX robustly enhanced Bcl- 2 inactivation by CAV-1 in MDA-MB-231 cells, while p53-mediated cell cycle arrest by DTX was more pronounced in CAV-1-low but p53-functional MCF-7 cells. In parallel with the data from breast cancer cell lines, CAV-1-transfected MCF-7 cells showed higher efficacy of DTX treatment in a xenograft model.

\section{Conclusion}

We clearly demonstrated cooperative effects between CAV-1 and DTX in mediating apoptosis, suggesting that the levels of CAV-1 expression might be an important indicator for DTX use in breast cancer.

\author{
Correspondence: Kyong Hwa Park, MD, PhD \\ Division of Oncology/Hematology, \\ Department of Internal Medicine, \\ Korea University College of Medicine, \\ 73 Inchon-ro, Seongbuk-gu, Seoul 02841, Korea \\ Tel: 82-2-920-6841 \\ Fax: 82-2-920-6520 \\ E-mail: khpark@korea.ac.kr \\ Received June 24, 2015 \\ Accepted August 13, 2015 \\ Published Online September 21, 2015
}

Key words

Caveolin 1, Breast neoplasms, Apoptosis, Docetaxel

\section{Introduction}

Caveolin-1 (CAV-1) is a member of the caveolin family of scaffold proteins that regulate the activity of many signaling molecules inside caveolae. Functionally, CAV-1 is implicated in various normal cell behaviors, including lipid transporta- tion, cell growth, and death regulation. In transformed cells, alterations of CAV-1 and its loss are associated with reduced invagination and a loss of caveolae [1]. Expression of CAV-1 in cancer cells is cell-type dependent; it is more likely to be downregulated in ovarian, colon, and mesenchymal sarcomas and upregulated in lung, bladder, esophageal, and pancreatic cancers [2]. In breast cancer, CAV-1 was initially 
considered a tumor suppressor based on the findings of low gene expression related to the mechanisms underlying the inactivating point mutation, silencing, or downregulation of CAV-1 in invasive cancers [3]. However, a growing body of evidence has shown that high levels of CAV-1 expression are more associated with basaloid cancers than estrogen receptor-negative and/or progesterone receptor-negative, or HER-2-expressing cancers [4]. In addition, CAV-1 expression has also been associated with aggressive cancer subtypes, such as inflammatory breast cancer, due to promoter hypomethylation [5]. Recent studies of the biologic activities of CAV-1 have focused more on its roles in cancer-associated fibroblasts. The protective roles of stromal CAV-1 against mammary hyperplasia and tumorigenesis in breast cancer have been demonstrated in multiple studies [6,7], and the loss of CAV-1 in stromal cells has been shown to be an independent prognostic factor in patients with early breast cancer $[2,8]$. These findings imply that the biological functions of CAV-1 might be multidimensional and cell-type dependent in breast cancer. However, the biological roles of CAV-1 in the epithelial compartment, particularly in the triple-negative breast cancer subtype, are largely unknown.

Taxanes are potent anticancer agents that function by binding to the $\beta$ subunits of tubulin, which results in stabilization of tubulin polymerization and repression of the dynamic instability of spindles. These activities lead to cell cycle arrest in the G2 / M phase. Docetaxel (DTX) and paclitaxel (PTX) are commonly used in the treatment of metastatic breast, lung, ovarian, and digestive cancers; however, DTX treatment has shown better clinical efficacy compared to PTX in patients with breast cancer [9]. The better affinity of DTX for tubulin and the subsequent induction of both apoptotic and necrotic cell death have been suggested as potential mechanisms for the superior antitumor activity of DTX compared to PTX. Clinical data have indicated that the addition of taxane to conventional adjuvant chemotherapy improves the prognosis of patients with early breast cancer, and these benefits are mainly observed in the triple-negative subgroup [10]. However, the use of taxanes is limited due to a variety of toxicities, including bone marrow suppression, arrhythmia, peripheral neuropathy, skin and nail changes, and gastrointestinal problems. Thus, identification of biomarkers that can predict the therapeutic effects of taxanes in breast cancer is an important unmet clinical need. Significant efforts have been made to identify potential predictive markers, including single microtubule-associated protein, tau [11], or panels of gene signatures [12]. However, there is no biomarker for DTX that can guide physicians in selecting the best treatment regimen.

A recent in vitro study showed that the specific phosphorylation of CAV-1 enhances the PTX-mediated cytotoxicity in MCF-7 cells, which are a luminal type of breast cancer cells
[13]. However, the potential association of CAV-1 and DTX response in various subtypes of breast cancer is not yet fully understood. Therefore, in this study, we attempted to determine whether CAV-1 functions as a modulator of cell growth and the cytotoxic activity of DTX in different subtypes of breast cancer cells in in vitro and in vivo models.

\section{Materials and Methods}

\section{Cell culture}

The ZR75-1, T47D, SKBR3, HCC1954, BT474, Hs578T, MDA-MB-231, MDA-MB-468, and MCF-7 cell lines were purchased from the American Type Culture Collection (ATCC, Manassas, VA). ZR75-1, T47D, SKBR-3, HCC1954, and MDA-MB-231 cells were maintained in RPMI-1640 medium (Thermo Fisher Scientific Inc., Waltham, MA). MCF7 cells were maintained in RPMI- 1640 medium supplemented with $4 \mathrm{mg} / \mathrm{mL}$ of insulin, human recombinant, zinc solution (Life Technologies, Grand Island, NY). BT474, Hs578T, and MDA-MB-468 cells were maintained in Dulbecco's modified Eagle's medium (Welgene Inc., Deagu, Korea). All of the culture media were supplemented with $10 \%$ fetal bovine serum, 100 units $/ \mathrm{mL}$ of penicillin, 100 $\mathrm{mg} / \mathrm{mL}$ of streptomycin, and $2 \mathrm{mM}$ of L-glutamine.

\section{Cell viability assay}

Cells were seeded at a density of $2 \times 10^{5}$ cells in 6-well plates. After 24 hours, the cells were treated with various concentrations of DTX. Quantitative measures of cell viability were determined using a 3-[4,5-dimethylthiazol-2-yl]-2, 5-diphenyltetrazolium (MTT) assay. Briefly, MTT solution was added to the plates after treatment with DTX, and the plates were incubated for another 4 hours at $37^{\circ} \mathrm{C}$. The absorbance of the converted MTT dye was measured at 540 nm using an iMARK microplate reader (Bio-Rad Laboratories Inc., Berkeley, CA). In all experiments the cell viability was expressed as a relative percentage of the untreated cells with error bars. All experiments were repeated at least twice. Statistical analyses were performed using Student's t tests. p-values less than 0.05 were considered significant.

\section{Western blot analyses}

The cells were harvested in lysis buffer $(20 \mathrm{mM}$ Tris- $\mathrm{HCl}$ [pH 7.5], 0.5\% Triton X-100, $150 \mathrm{mM}$ sodium chloride, 10\% glycerol, $1 \mathrm{mM}$ sodium orthovanadate, $20 \mathrm{mM}$ sodium fluoride, and $100 \mathrm{mM}$ phenylmethylsulfonyl fluoride) containing 
Xpert protease inhibitor cocktail solution (genDEPOT Inc., Barker, TX) and incubated on ice for $50 \mathrm{~min}$. Proteins $(30 \mu \mathrm{g})$ were separated on $8 \%$ or $12 \%$ sodium dodecyl sulfate-polyacrylamide gel and transferred to polyvinylidene fluoride membranes. The membranes were blocked in $5 \%$ nonfat milk for 1 hour at room temperature and incubated with the appropriate primary antibodies at $4^{\circ} \mathrm{C}$ overnight, followed by washing and incubation with horseradish peroxidaseconjugated secondary antibodies. The protein bands were visualized using the ECL system (GE Healthcare, Piscataway, NJ), and the images were developed on X-ray film (Agfa HealthCare NV, Mortsel, Belgium). Antibodies for the estrogen receptor, ErbB2, p53, Bcl-2, and Bax were obtained from Santa Cruz Biotechnology Inc. (Santa Cruz, CA). Antibodies for CAV-1, poly-ADP-ribose polymerase, and pBcl-2 were purchased from Cell Signaling Technology Inc. (Danvers, MA). $\beta$-Actin was obtained from Sigma-Aldrich Co. LLC (St. Louis, MO).

\section{4. $\left[{ }^{3} \mathrm{H}\right]$ thymidine incorporation assay}

DNA synthesis was measured using a $\left[{ }^{3} \mathrm{H}\right]$ thymidine uptake assay. The cells were plated in 6-well plates, serum starved for 48 hours, and then treated with DTX for 48 hours. In the last 4 hours, the cells were pulsed with $1 \mu \mathrm{Ci} / \mathrm{mL}$ $\left[{ }^{3} \mathrm{H}\right]$ thymidine (GE Healthcare, Milano, Italy). The cells were washed twice with serum-free medium and precipitated with 5\% trichloroacetic acid for 15 minutes at $4^{\circ} \mathrm{C}$. The precipitates were washed twice with $95 \%$ ethanol, dissolved in $1 \mathrm{~mL}$ of $\mathrm{NaOH}$, and then analyzed by liquid scintillation counting.

\section{CAV-1 stable transfection}

A pcDNA3.1 expression plasmid containing CAV-1 cDNA was obtained from Dr. Hong-Guang Zhu (Fudong University, Shanghai, China). MCF-7 and ZR75-1 cells were stably transfected with a CAV-1 expression plasmid (CAV-1) or empty vector (EV) with Lipofectamine 2000 reagent (Life Technologies). Forty-eight hours after the transfection, the media was replaced with G418-containing media (400 $\mu \mathrm{g} / \mathrm{mL}$ ). Individual colonies were selected during the subsequent 2 weeks of selection, and positive transfectants were screened by western blot analyses.

\section{6. siRNA transfection}

siRNA targeting CAV-1 (sense, 5'-AGACGAGCUGAGCGAGAAGCA-3'; antisense, 5'-UGCUUCUCGCUCAGCUCG UCU-3'; Lot No. S20121127-020) was purchased from Bioneer Corporation (Daejeon, Korea). MDA-MB-231 and Hs578T cells were seeded and transiently transfected with the CAV-
1 expression plasmid or EV with Lipofectamine 2000 reagent (Life Technologies). After transfection, the downregulation of CAV-1 expression was confirmed by western blot analyses.

\section{Cell cycle analysis}

Harvested cells were fixed in 70\% ice-cold ethanol and incubated with $4 \mu \mathrm{g} / \mathrm{mL}$ of propidium iodide and $100 \mu \mathrm{g} / \mathrm{mL}$ of RNase A for 45 minutes at $37^{\circ} \mathrm{C}$. Stained samples were analyzed using FACScalibur and Cell Quest Pro (BD, Oxford, UK).

\section{Apoptosis assay}

Apoptosis was evaluated using annexin V-fluorescein isothiocyanate (annexin V-FITC; BD Biosciences, San Jose, CA). Briefly, cells were seeded and treated with DTX (10 nM) for 24 hours. The cells were then harvested and stained with $5 \mu \mathrm{L}$ of annexin V-FITC and $5 \mu \mathrm{L}$ of propidium iodide at room temperature for 15 minutes. The stained samples were analyzed by FACScalibur and Cell Quest Pro (BD).

\section{In vivo breast cancer xenograft assays}

Female nude mice (BALB/c, nu/nu), aged 6 weeks and weighing 18-22 g, were purchased from Orient Bio Inc. (Seongnam, Korea). All mice were implanted with $0.72 \mathrm{mg}$ of 60 -day release $17 \beta$-estradiol pellets $(2.5 \mathrm{mg} / \mathrm{p}$, Innovative Research of America, Sarasota, FL). MCF-7 $\left(5 \times 10^{6}\right)$ cells were implanted subcutaneously into mice ( $\mathrm{n}=5$ per group). Seven days later, EV / MCF-7 and CAV-1 / MCF-7 cells ( $5 \times 10^{6}$ cells) in $100 \mu \mathrm{L}$ of phosphate buffered saline (PBS) were injected subcutaneously into the right flank of the mice. When the tumor reached a size greater than $200 \mathrm{~mm}^{3}$, the mice were treated with PBS or DTX at $10 \mathrm{mg} / \mathrm{kg}$ twice a week in the four following randomly divided groups (5 mice per group): EV / MCF-7 (PBS control and DTX) and CAV-1/MCF-7 (PBS control and DTX). The tumor diameters were measured using calipers twice per week for more than 3 weeks. The tumor volumes were calculated using the following formula: Volume $\left(\mathrm{mm}^{3}\right)=W \mathrm{Widh}^{2} \times$ Length $/ 2$. The mice were then sacrificed, and the tumor tissues were collected for histological analyses. Differences in tumor growth between the animal treatment groups were analyzed using two-way repeated measures analysis of variance (ver. 10.0, SPSS Inc., Chicago, IL). In all cases, p-values less than 0.05 were considered significant. All animal procedures were performed according to the Institutional Animal Care and Use and Institutional Review Board committee (KIRAMS2011-20) at the Korea Institute of Radiological and Medical Science (KIRAMS, Seoul, Korea). 


\section{Histopathologic analysis}

Expression of CAV-1 (Santa Cruz Biotechnology Inc.), Ki67 (Dako North America Inc., Carpinteria, CA), and p53 (DO-7, Leica Biosystems Newcastle Ltd., Newcastle upon Tyne, UK) was determined using an immunohistochemical approach on formalin-fixed paraffin-embedded 5- $\mu$ m-thick tissue sections. For each case, one representative section was stained with hematoxylin and eosin.

\section{Results}

\section{Correlation of CAV-1 expression and DTX sensitivity in three subtypes of breast cancer cells}

To investigate the potential relationship between CAV-1 and DTX sensitivity in three representative subtypes (estrogen receptor-positive, ErbB2-positive, triple negative) of breast cancer cells, we first profiled the levels of expression of CAV-1 in a panel of nine different breast cancer cell lines (ZR75-1, T47D, MCF-7, SKBR3, HCC1954, BT474, Hs578T, MDA-MB-231, and MDA-MB-468). As shown in Fig. 1A, CAV-1 expression was detected in two of the triple-negative

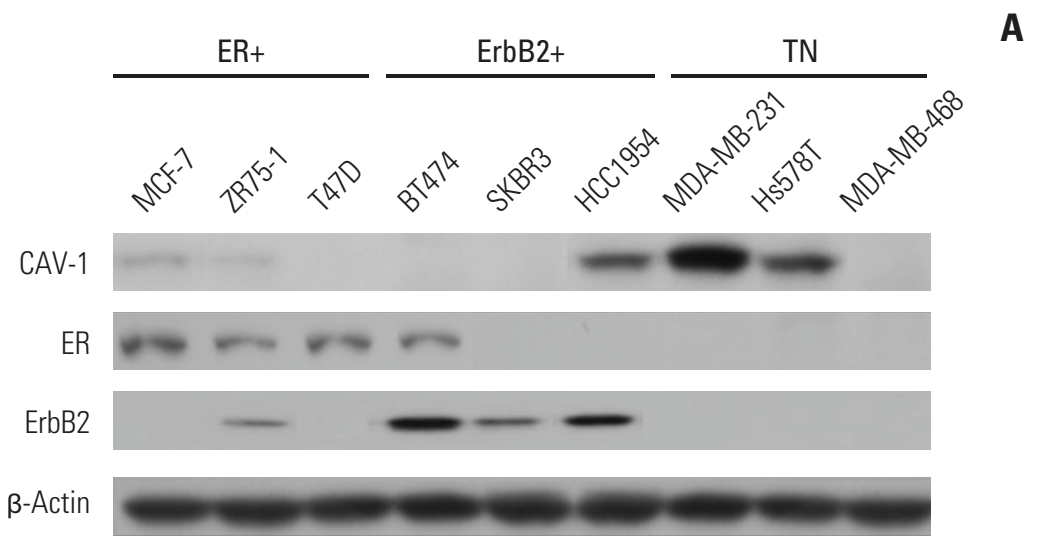

B

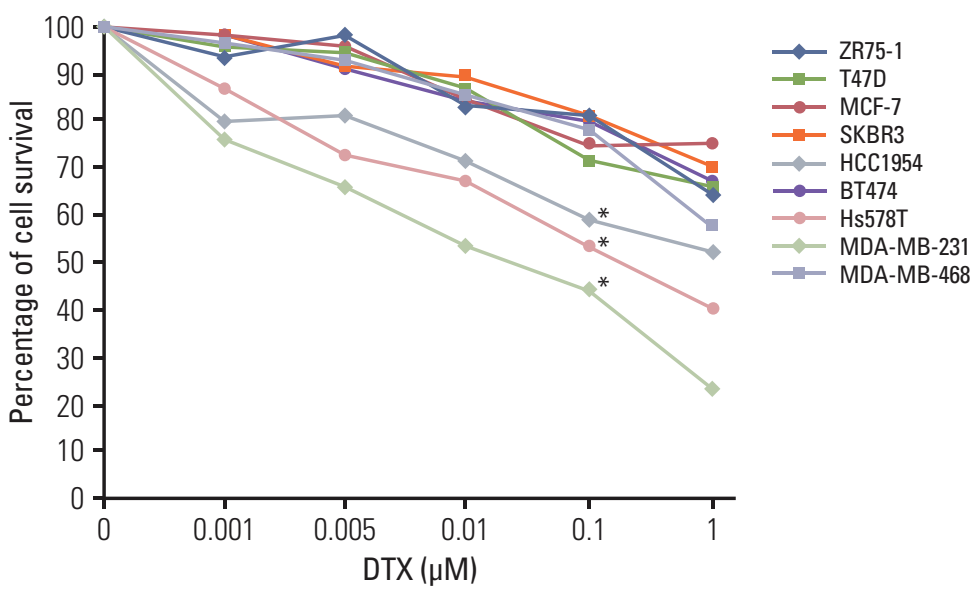

Fig. 1. Caveolin-1 (CAV-1)-expressing breast cancer cells are more sensitive to docetaxel (DTX). (A) The levels of expression of the estrogen receptor (ER), ErbB-2, and CAV-1 were evaluated by western blot analyses according to subtype of breast cancer cells. $\beta$-Actin was used as an internal control. (B) ZR75-1, T47D, MCF-7, SKBR3, HCC1954, BT474, Hs578T, MDAMB-231, and MDA-MB-468 cells were treated with the indicated concentrations of DTX, and the mean percentage of survival in the MTT assay is shown. TN, triple negative; MTT, 3-[4,5-dimethylthiazol-2-yl]-2,5-diphenyltetrazolium. * $\mathrm{p}<0.05$ compared to MCF-7 cells. 
A

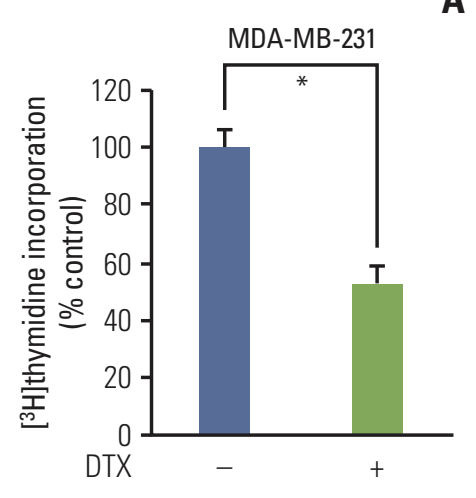

B

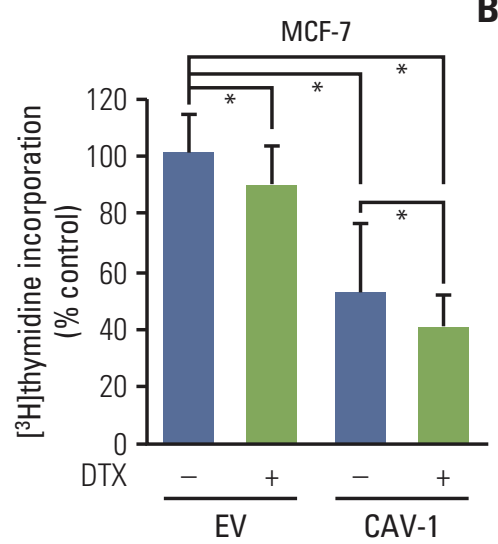

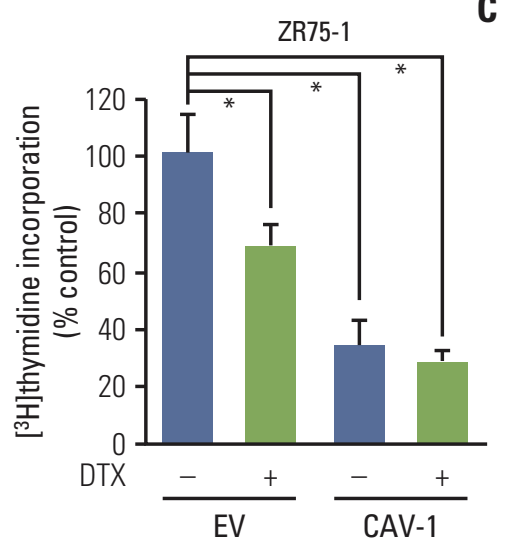

Fig. 2. Caveolin-1 (CAV-1) enhances the antiproliferative activity of docetaxel (DTX). Associations between the status of CAV-1 expression and the DTX-induced antiproliferative activity were analyzed by $\left[{ }^{3} \mathrm{H}\right]$ thymidine incorporation in MDAMB-231 cells (A), MCF-7 cells (B), and ZR75-1 cells (C) after transfection with an empty vector (EV) or a CAV-1 expression vector $(\mathrm{CAV}-1)$, respectively. The bars show the mean value of $\left[{ }^{3} \mathrm{H}\right]$ thymidine incorporation, and the error bars indicate standard deviation. ${ }^{*} \mathrm{p}<0.05$ in Student's t tests.

cell lines (MDA-MB-231 and Hs578T) and one of the ErbB2positive cell lines (HCC1954), while it was downregulated in other breast cancer cell lines. Next, to determine whether DTX sensitivity differed according to the levels of CAV-1 expression, the cell survival rate after DTX treatment was analyzed in these cell lines. As shown in Fig. 1B, the percentage of viable cells in the CAV-1-expressing cell lines (MDAMB-231, Hs578T, and HCC1954) was significantly decreased after DTX treatment in a dose-dependent manner compared to that in other breast cancer cell lines $(p<0.05)$. These results suggested that CAV-1 might have a modulating role in DTXinduced cell death in breast cancer cells.

\section{Effects of CAV-1 on the antiproliferative activity of DTX}

To elucidate the biological effects of CAV-1 in the antiproliferative activities of DTX, we examined the changes in thymidine incorporation after DTX treatment in breast cancer cell lines with or without CAV-1 expression. As shown in Fig. 2A, CAV-1-expressing MDA-MB-231 cells showed significantly decreased thymidine incorporation after DTX treatment $(\mathrm{p}<0.05)$. When the low CAV-1-expressing MCF7 and ZR75-1 cells were transfected by either the CAV-1 gene or a control vector, CAV-1-transfected cells showed an obvious decrease in thymidine incorporation compared to control vector-transfected cells (Fig. 2B and C). In addition, the antiproliferative effects of DTX were greatly enhanced by CAV-1 overexpression, and this finding was consistent in both MCF-7 and ZR75-1 cells. These findings indicated that
CAV-1 might act as an antiproliferative factor and a modulator of DTX activity in breast cancer cells.

\section{CAV-1 increased DTX-induced G2/M cell cycle arrest}

The antiproliferative activities of DTX mainly result from its inhibitory effects on mitotic cell division between metaphase and anaphase, which stabilizes microtubule assembly and prevents physiological microtubule depolymerization/disassembly [14]. To determine whether CAV-1 altered DTX-induced cell cycle arrest, we examined the changes in the cell cycle that occurred after DTX treatment in MDA-MB-231 and MCF-7 cells with or without CAV-1 expression. As shown in Fig. 3A and B, G2/M arrest was expectedly induced after DTX treatment in both MDAMB-231 and MCF-7 cells. In addition, significantly higher percentages of cells arrested in the G2/M phases after DTX treatment were observed in CAV-1-transfected MCF-7 cells compared to EV-transfected MCF-7 cells (35.7\% and 47.2\%, respectively, $\mathrm{p}<0.05)$. We then analyzed the levels of expression of cell cycle-related proteins in these cell lines after DTX treatment. In MDA-MB-231 cells, p21, p27, and cyclin A were increased, but the expression of cyclin D2 was decreased 48 hours after DTX treatment (Fig. 3C). CAV-1-transfected MCF-7 cells showed relatively enhanced expression levels of p21 and p27 and expression levels of cyclin D2 were downregulated compared to EV-transfected MCF-7 cells after DTX treatment (Fig. 3D). In addition, the expression level of cyclin B1 showed a greater increase after DTX treatment in CAV- 

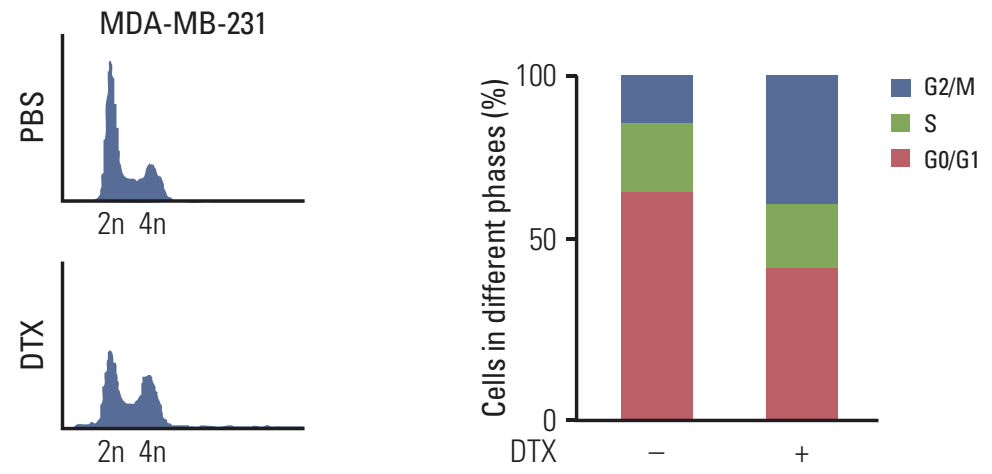

B
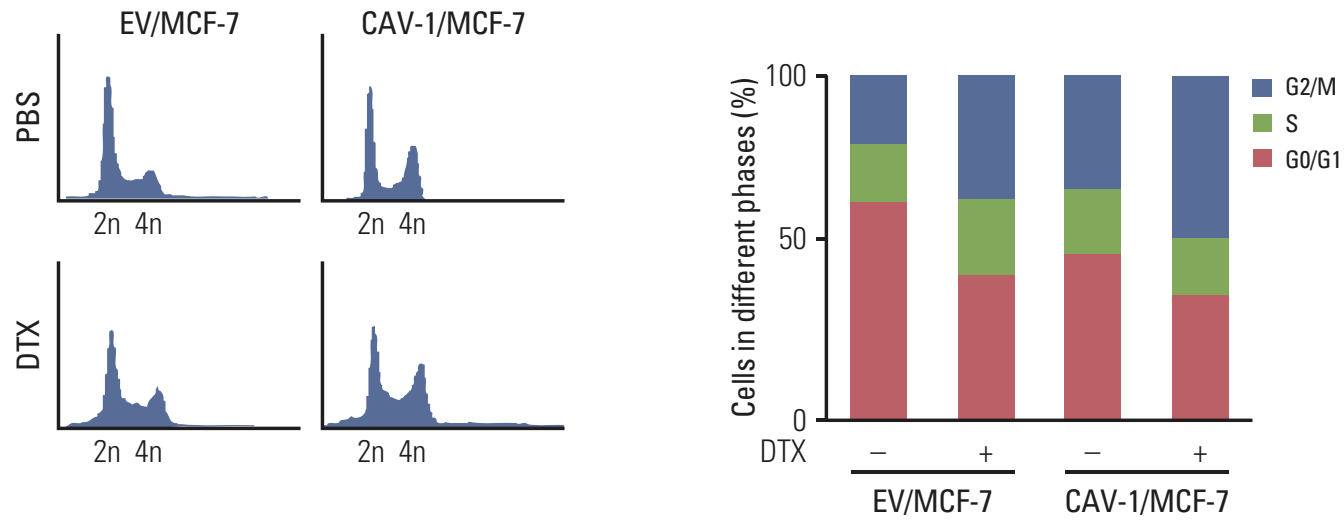

C
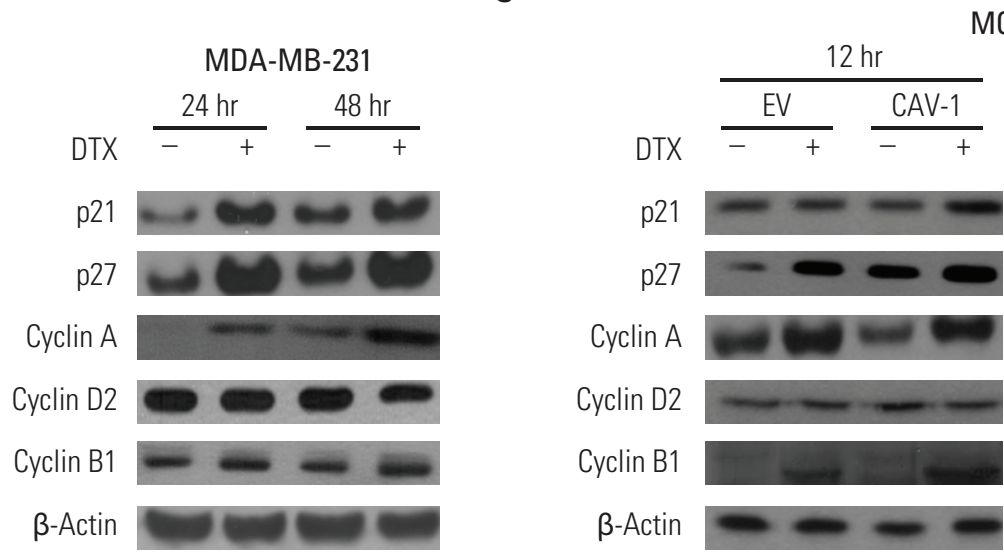

MCF-7
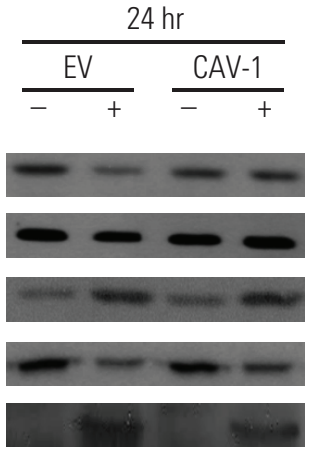

$-\infty$

Fig. 3. Caveolin-1 (CAV-1) induces cell cycle arrest in the G2/M phase. Changes in the cell cycle were analyzed with propidium iodide staining in MDA-MB-231 cells (A) and CAV-1-transfected MCF-7 (CAV-1/MCF-7) or empty vector-transfected MCF-7 (EV/MCF-7) cells (B). The bars show the mean percentages of the different phases of the cell cycle. Changes in cell cycle-related proteins were examined by western blot analyses in MDA-MB-231 (C) and CAV-1/MCF-7 or EV / MCF7 (D) cells. $\beta$-Actin was used as an internal control. PBS, phosphate buffered saline; DTX, docetaxel. 


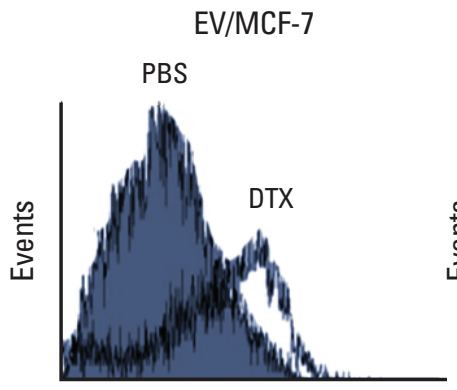

FL1-H
CAV/MCF-7

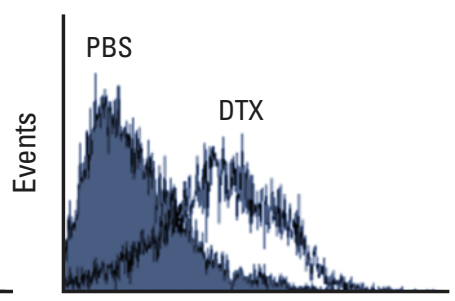

FL1-H

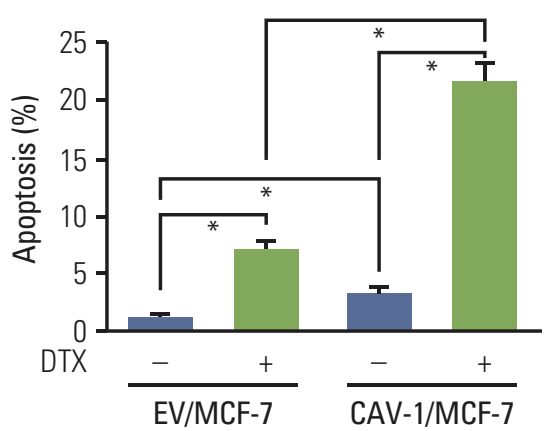

B

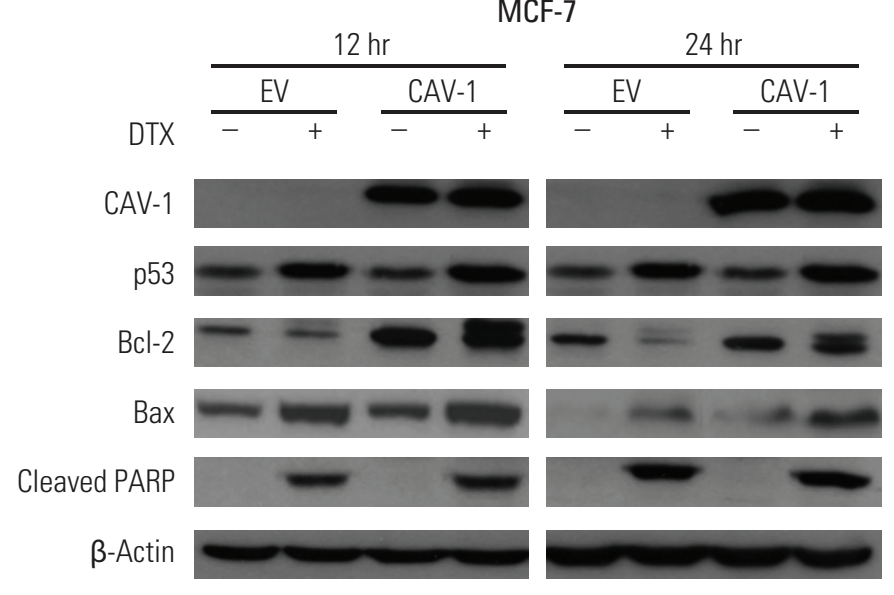

Fig. 4. Caveolin-1 (CAV-1) expression increases docetaxel (DTX)-induced apoptotic cell death. (A) Changes in the apoptotic cell population were evaluated by annexin V staining in CAV-1-transfected MCF-7 (CAV-1/MCF-7) or empty vector-transfected MCF-7 (EV / MCF-7) cells. The bars show the mean percentage of annexin V-positive cells. (B) Changes in the expression of apoptosis-related proteins were examined by western blot analyses in CAV-1/MCF-7 or EV/MCF-7 cells. $\beta$-Actin was used as an internal control. PBS, phosphate buffered saline; PARP, poly(ADP-ribose) polymerase. ${ }^{*} \mathrm{p}<0.05$ in Student's $\mathrm{t}$ tests.

1-transfected cells compared to that in EV-transfected cells. These results suggested that CAV-1 expression contributed to the DTX-induced G2/M cell cycle arrest in breast cancer cells.

\section{CAV-1 modulated DTX-induced apoptotic cell death}

To investigate the potential effects of CAV-1 in DTXinduced apoptotic cell death in breast cancer cells, the percentage change in apoptotic cell death was analyzed with annexin V-positive cells after DTX treatment. As shown in Fig. 4A, the calculated percentage of apoptotic cells induced by DTX was highly increased in CAV-1-transfected MCF-7 cells compared to EV-transfected MCF-7 cells (17.8\% vs. $4.2 \%$, respectively; $\mathrm{p}<0.05)$. In addition, the levels of $\mathrm{p} 53$,
Bax, and cleaved poly-ADP-ribose polymerase showed a greater increase after DTX treatment in CAV-1-transfected MCF-7 cells compared to EV-transfected MCF-7 cells (Fig. 4B). Unexpectedly, we found that the levels of Bcl-2, an antiapoptotic protein, were highly upregulated in CAV1-transfected MCF-7 cells compared to EV-transfected cells. In addition, the increased levels of Bcl-2 expression observed in CAV-1-transfected MCF-7 cells after DTX treatment were detected as two separate bands, which represented the phosphorylated and inactivated form of Bcl-2 protein, as previously reported [15].

Next, determine whether the mechanisms for this DTXinduced apoptotic cell death were affected by the endogenous expression levels of CAV-1, we assessed the total and phosphorylated forms of Bcl-2 protein after DTX treatment 
A
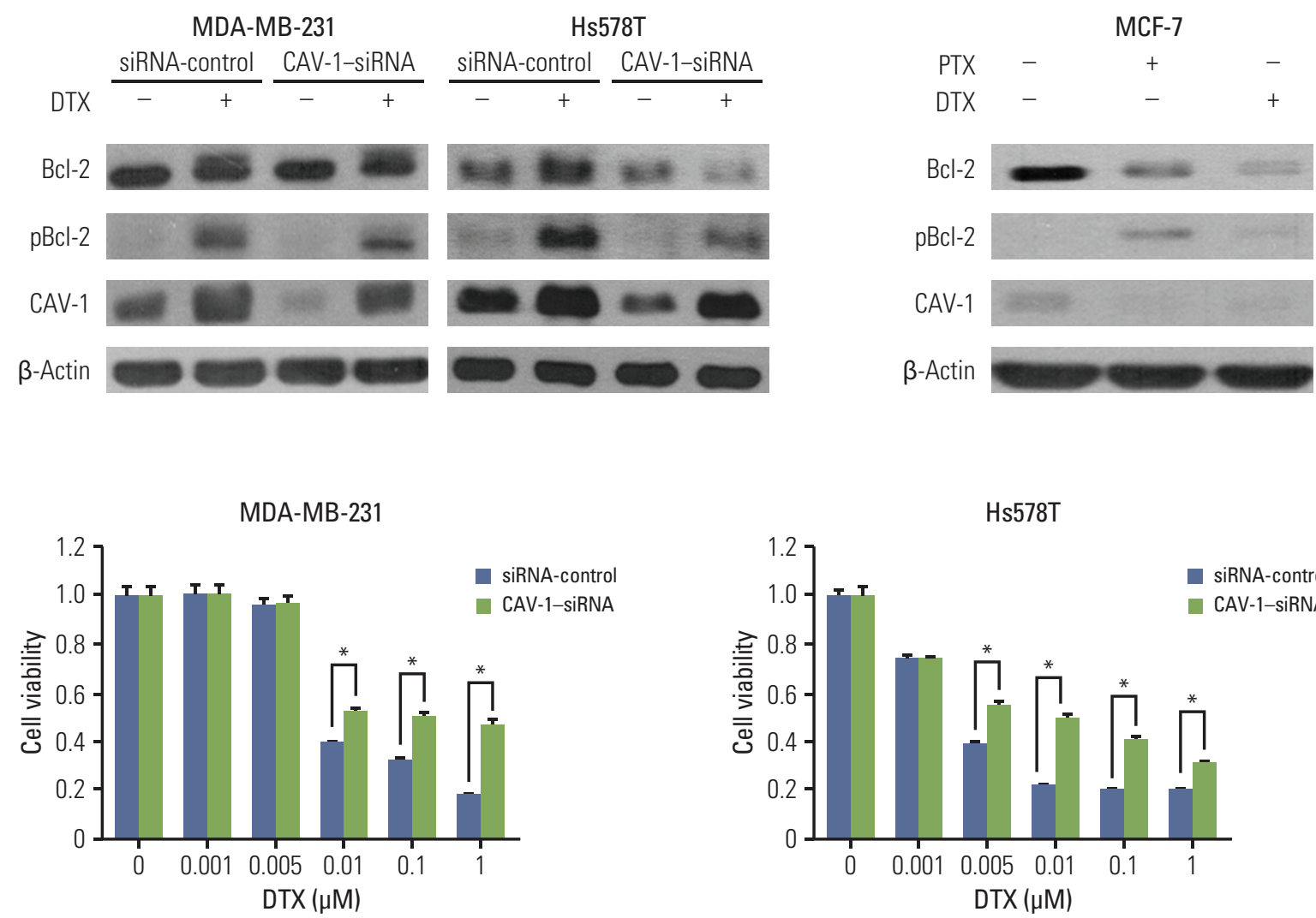

Fig. 5. Silencing of caveolin-1 (CAV-1) expression dampened the docetaxel (DTX)-induced phosphorylation of Bcl-2 and cytotoxicity in triple-negative breast cancer cells. The phosphorylation status was evaluated by western blot analyses in CAV-1 siRNA-transfected MDA-MB-231 (A) and MCF-7 (B) cells. $\beta$-Actin was used as an internal control. The mean percentages of survival in the MTT assay in CAV-1 siRNA-transfected MDA-MB-231 and Hs578T cells are shown (C). PTX, paclitaxel. ${ }^{*} \mathrm{p}<0.05$ compared to siRNA controls.

in MDA-MB-231 and MCF-7 cells. As shown in Fig. 5A, two triple-negative breast cancer cell types (MDA-MB-231 and Hs578T) with high levels of endogenous CAV-1 expression showed increased CAV-1 expression and the induction of Bcl-2 phosphorylation after DTX treatment. However, the changes in the levels of CAV-1 expression and phosphorylated $\mathrm{Bcl}-2$ were diminished after $\mathrm{CAV}-1$ siRNA transfection. In contrast, MCF-7 cells with very low levels of CAV-1 showed decreased Bcl-2 expression after DTX treatment, and CAV-1 expression was not induced (Fig. 5B). In addition, the silencing of CAV-1 resulted in significantly decreased DTXinduced cytotoxicity in two triple-negative breast cancer cells (Fig. 5C). Therefore, these results suggested that the endogenous expression of the CAV-1 gene plays a pivotal role in DTX-induced apoptosis in breast cancer cells.

\section{CAV-1 enhanced the antitumor effects of DTX in a breast cancer in vivo model}

The findings observed in the in vitro study were further evaluated in nude mice. The mean tumor size in each group of mice showed that the tumors of EV/MCF-7 cells treated with PBS showed the most rapid growth, followed by tumors of EV / MCF-7 cells treated with DTX. Notably, CAV-1-overexpressing MCF-7 cells (CAV-1/MCF-7) showed greatly attenuated tumor growth compared to that in EV/MCF-7 cells, and these growth-inhibitory effects were further enhanced by DTX treatment ( $<$ 0.001) (Fig. 6A). Immunohistochemical analyses were performed to examine the biological changes in the in vivo tumors (Fig. 6B). The protein expression of p53 was induced both by DTX treatment and by the ectopic expression of CAV-1 in MCF-7 cells, and its expression was further enhanced by the combinatorial effects 

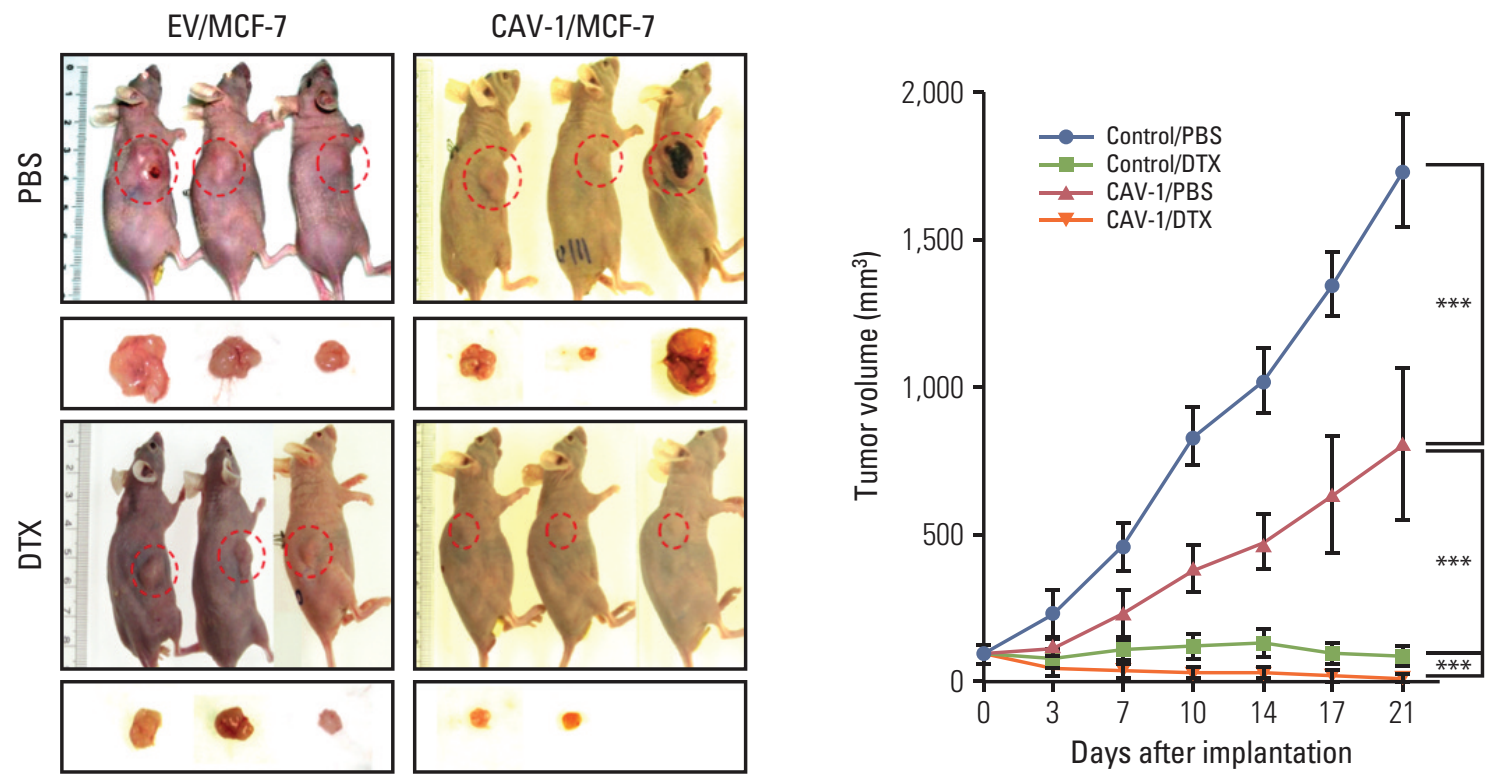

A

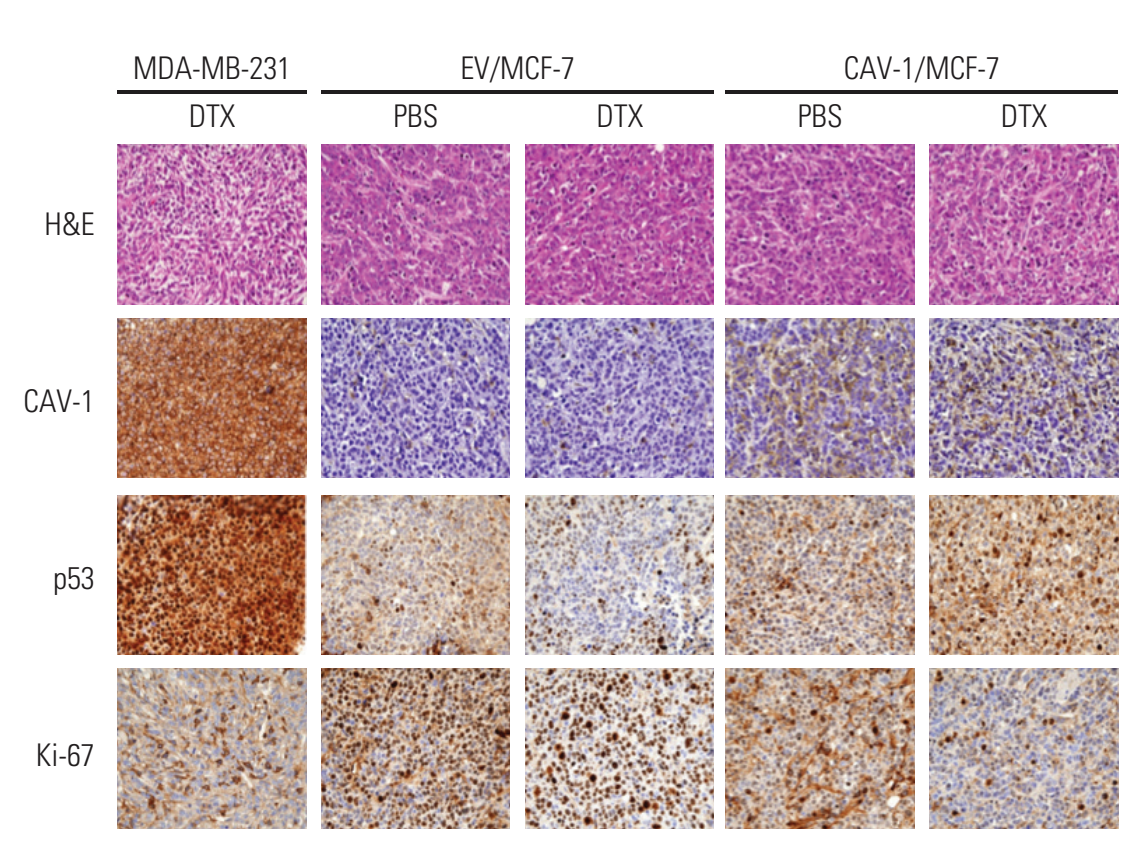

Fig. 6. Caveolin-1 (CAV-1) enhances docetaxel (DTX)-induced inhibition of tumor growth in vivo. (A) Images of the mice, tumors, and the mean tumor volume from each group: empty vector-transfected MCF-7 (EV / MCF-7) (phosphate buffered saline [PBS] control and DTX) and CAV-1-transfected MCF-7 (CAV-1/MCF-7) (PBS control and DTX). The error bars represent the standard error. ${ }^{* * *} \mathrm{p}<0.001$. (B) Representative staining results from hematoxylin and eosin (H\&E) and immunohistochemical staining for CAV-1, p53, and Ki-67 in tissues isolated from EV/MCF-7 or CAV-1/MCF-7 cells. Tissues isolated from mice induced by MDA-MB-231 cells were used as a control for the expression of these proteins. 
of DTX and CAV-1. Consequently, the immunostaining of Ki-67 was more decreased in DTX-treated and CAV-1-transfected MCF-7 tumors compared to DTX-treated EV/MCF-7 tumors. Further antiproliferative effects of DTX in the presence of CAV-1 expression in MCF-7 cells were confirmed by Ki-67 staining.

\section{Discussion}

The current study addressed the question of whether CAV-1 had tumor-suppressive functions and activity in DTX-mediated cytotoxicity in different subtypes of breast cancer cells. The main findings of this study were that CAV1 had antiproliferative and cell cycle arrest functions and modulated the DTX-induced apoptosis of MDA-MB-231 and MCF-7 breast cancer cells, which were confirmed in vivo.

CAV-1 has been suggested to function either as a tumor suppressor or as an oncogene depending on the tumor type and context of tumor progression. In breast cancer, oncogenic functions of CAV-1 have been proposed based on the findings of its upregulation in highly aggressive drug-resistant subsets of basal-like, metaplastic, or inflammatory cancers [16]. While its prognostic role is unclear, in a comprehensive meta-analysis, the epithelial expression of CAV-1 showed significant positive correlations with the clinical characteristics of triple negativity and positivity for cytokeratin $5 / 6$ [17]. More recently, overexpression of CAV- 1 was observed in the claudin-low mesenchymal subtype of breast cancer cells in murine models, implicating it as a possible biomarker for this challenging subgroup [18]. However, these observations have not been clearly supported by biologic studies in any triple-negative breast cancer model. In the current study, CAV-1 was highly expressed in two triple-negative and one ErbB2 subtype of breast cancer cell lines in our panel (Fig. 1A). In addition, ectopic expression of CAV-1 decreased cell proliferation by inducing cell cycle arrest in the G2/M phase in MCF-7 cells (Fig. 2). Of note, CAV-1 per se had efficacy similar to that of DTX in MCF-7 cells, which supported the findings of earlier studies reporting that CAV- 1 is a tumor suppressor in human breast cancer cells [19]. Therefore, determine the clinical relevance of CAV-1 in the triplenegative mesenchymal subtype of breast cancer is warranted.

The phosphorylated form of CAV-1 is known to enhance PTX-mediated cytotoxicity in MCF-7 cells. Shajahan et al. suggested that CAV-1 sensitizes apoptosis by the induction of p21 in a p53-dependent manner and by suppression of Bcl2. In reality, however, CAV-1 expression is observed mainly in the basal type of tumors, which contain nonfunctional p53 protein. Indeed, in this study, the CAV-1-expressing cell lines that showed high sensitivity to DTX (HCC1954, MDAMB-231, and Hs578T) are known to have mutant p53 [20]. Thus, in tumor cells with nonfunctional p53, different mechanisms might contribute to CAV-1 in taxane-mediated cytotoxicity. More recently, data have emerged suggesting that Bcl-2 plays an important role in PTX-mediated cytotoxicity. Although the precise molecular mechanism for regulating the expression levels of Bcl-2 family proteins is not clear, a study by Shajahan et al. [15] expanded prior understanding of the activity of CAV-1 by suggesting that the phosphorylated form of CAV- $1 \alpha$ facilitates PTX-induced apoptosis by inactivating $\mathrm{Bcl}-2$ and $\mathrm{Bcl}-\mathrm{xL}$. In the current study, dramatically increased levels of the phosphorylated form of Bcl-2 were observed in CAV-1 transfected MCF-7 cells compared to MCF-7 cells, which showed an increase in p53 protein rather than phosphorylated Bcl-2 after DTX treatment (Fig. 4). Conversely, the knock down of CAV-1 in MDA-MB231 and Hs578T cells resulted in a significant decrease in the phosphorylated form of Bcl-2 after DTX treatment (Fig. 5A). These data suggested that the modulating effects of CAV-1 in DTX-induced apoptosis resulted mainly from the inhibitory activity on Bcl-2 in p53 nonfunctional MDAMB-231 and Hs578T cells.

DTX is known to have a higher affinity for microtubules than PTX due to its more potent induction of Bcl-2 phosphorylation [14]. While DTX is one of the most commonly used chemotherapeutics in patients with breast cancer, predictive biomarkers of DTX sensitivity are still inconclusive [21]. Based on in vitro data, CAV-1 has been suggested as a sensitizer of PTX in its induction of apoptosis in estrogen receptor-positive breast cancer cells [13]. In the current study, unlike other triple-negative cell line, CAV-1-negative MDAMB-468 cells showed very low sensitivity to DTX, and CAV1-positive HCC1954 cells were significantly more sensitive to DTX than the other ErbB2 type of cells. These results suggested that CAV-1 might be an important determinant of DTX sensitivity in breast cancer. In our in vitro study, transfection of CAV-1 significantly dampened the proliferation of tumor cells, and its effects were enhanced by DTX treatment (Fig. 2). This was further confirmed by the knock down of CAV-1 in MDA-MB-231 and Hs578T cells, which resulted in significantly decreased DTX-induced cytotoxicity (Fig. 5C). Consistent with these results, in the in vivo study, immunohistochemical staining of the tumors showed a further increase in p53 expression and a decrease in the proliferative marker, Ki-67, in CAV-1-transfected MCF-7 cells treated with DTX (Fig. 6B). The data presented here confirmed that CAV-1, a highly versatile and context-dependent gene, had tumor-suppressive and chemomodulating effects in breast cancer cells in vivo. Given that CAV-1 is more preferentially expressed in the clinically challenging subtypes of breast can- 
cers, including the basal, inflammatory, and metaplastic subtypes, it should be evaluated as a biomarker for prediction of response to DTX.

Our data suggesting the chemomodulating effects of CAV-1 in breast cancer cells were in contrast to the findings of previous reports that CAV-1 is a mediator of multidrug resistance [22-24]. Data addressing the relationship of CAV-1 and drug resistance was first reported from studies of adriamycin-resistant MCF-7 cells [22] and taxol-resistant A549 cells [25]. However clinical data from studies of patients with breast cancer, did not support the in vitro data. CAV-1 expression was not a prognostic factor in patients with breast cancer treated with anthracycline-based adjuvant treatment [4]. In a small series of patients with advanced non-small-cell lung cancer, positive expression of CAV-1 was a predictive factor of poor response to gemcitabine-based chemotherapy and worse clinical outcomes [24]. Data on the clinical significance of CAV-1 relevant to responses to chemotherapy and prognoses are still limited, therefore our data should be confirmed in a clinical study of taxane-treated patients with other subtypes of cancer.

\section{Conclusion}

Taken together, our data suggested that CAV-1 has tumorsuppressive functions as well as modulating activities in DTX-induced apoptosis of breast cancer cells through different mechanisms.

\section{Conflicts of Interest}

Conflict of interest relevant to this article was not reported.

\section{Acknowledgments}

This study was financially supported by a Korea University Research Fund.

\section{References}

1. Koleske AJ, Baltimore D, Lisanti MP. Reduction of caveolin and caveolae in oncogenically transformed cells. Proc Natl Acad Sci U S A. 1995;92:1381-5.

2. Sotgia F, Martinez-Outschoorn UE, Howell A, Pestell RG, Pavlides S, Lisanti MP. Caveolin-1 and cancer metabolism in the tumor microenvironment: markers, models, and mechanisms. Annu Rev Pathol. 2012;7:423-67.

3. Park SS, Kim JE, Kim YA, Kim YC, Kim SW. Caveolin-1 is down-regulated and inversely correlated with HER2 and EGFR expression status in invasive ductal carcinoma of the breast. Histopathology. 2005;47:625-30.

4. Savage K, Lambros MB, Robertson D, Jones RL, Jones C, Mackay A, et al. Caveolin 1 is overexpressed and amplified in a subset of basal-like and metaplastic breast carcinomas: a morphologic, ultrastructural, immunohistochemical, and in situ hybridization analysis. Clin Cancer Res. 2007;13:90-101.

5. Rao X, Evans J, Chae H, Pilrose J, Kim S, Yan P, et al. CpG island shore methylation regulates caveolin-1 expression in breast cancer. Oncogene. 2013;32:4519-28.

6. Lisanti MP, Martinez-Outschoorn UE, Sotgia F. Oncogenes induce the cancer-associated fibroblast phenotype: metabolic symbiosis and "fibroblast addiction" are new therapeutic targets for drug discovery. Cell Cycle. 2013;12:2723-32.

7. Martins D, Beca FF, Sousa B, Baltazar F, Paredes J, Schmitt F. Loss of caveolin-1 and gain of MCT4 expression in the tumor stroma: key events in the progression from an in situ to an invasive breast carcinoma. Cell Cycle. 2013;12:2684-90.
8. Simpkins SA, Hanby AM, Holliday DL, Speirs V. Clinical and functional significance of loss of caveolin-1 expression in breast cancer-associated fibroblasts. J Pathol. 2012;227:490-8.

9. Lyseng-Williamson KA, Fenton C. Docetaxel: a review of its use in metastatic breast cancer. Drugs. 2005;65:2513-31.

10. Martin M, Rodriguez-Lescure A, Ruiz A, Alba E, Calvo L, Ruiz-Borrego M, et al. Molecular predictors of efficacy of adjuvant weekly paclitaxel in early breast cancer. Breast Cancer Res Treat. 2010;123:149-57.

11. Pusztai L, Jeong JH, Gong Y, Ross JS, Kim C, Paik S, et al. Evaluation of microtubule-associated protein-Tau expression as a prognostic and predictive marker in the NSABP-B 28 randomized clinical trial. J Clin Oncol. 2009;27:4287-92.

12. Tabchy A, Valero V, Vidaurre T, Lluch A, Gomez H, Martin $\mathrm{M}$, et al. Evaluation of a 30-gene paclitaxel, fluorouracil, doxorubicin, and cyclophosphamide chemotherapy response predictor in a multicenter randomized trial in breast cancer. Clin Cancer Res. 2010;16:5351-61.

13. Shajahan AN, Wang A, Decker M, Minshall RD, Liu MC, Clarke R. Caveolin-1 tyrosine phosphorylation enhances paclitaxel-mediated cytotoxicity. J Biol Chem. 2007;282:5934-43.

14. Gligorov J, Lotz JP. Preclinical pharmacology of the taxanes: implications of the differences. Oncologist. 2004;9 Suppl 2:3-8.

15. Shajahan AN, Dobbin ZC, Hickman FE, Dakshanamurthy S, Clarke R. Tyrosine-phosphorylated caveolin-1 (Tyr-14) increases sensitivity to paclitaxel by inhibiting BCL2 and BCLxL proteins via c-Jun N-terminal kinase (JNK). J Biol 
Chem. 2012;287:17682-92.

16. Van den Eynden GG, Van Laere SJ, Van der Auwera I, Merajver SD, Van Marck EA, van Dam P, et al. Overexpression of caveolin-1 and -2 in cell lines and in human samples of inflammatory breast cancer. Breast Cancer Res Treat. 2006;95:219-28.

17. Ma X, Liu L, Nie W, Li Y, Zhang B, Zhang J, et al. Prognostic role of caveolin in breast cancer: a meta-analysis. Breast. 2013; 22:462-9.

18. Thompson DE, Siwicky MD, Moorehead RA. Caveolin-1 expression is elevated in claudin-low mammary tumor cells. Cancer Cell Int. 2012;12:6.

19. Williams TM, Medina F, Badano I, Hazan RB, Hutchinson J, Muller WJ, et al. Caveolin-1 gene disruption promotes mammary tumorigenesis and dramatically enhances lung metastasis in vivo. Role of Cav-1 in cell invasiveness and matrix metalloproteinase (MMP-2/9) secretion. J Biol Chem. 2004; 279:51630-46.

20. Haldar S, Chintapalli J, Croce CM. Taxol induces bcl-2 phosphorylation and death of prostate cancer cells. Cancer Res. 1996;56:1253-5.
21. Singel SM, Cornelius C, Batten K, Fasciani G, Wright WE, Lum $\mathrm{L}$, et al. A targeted RNAi screen of the breast cancer genome identifies KIF14 and TLN1 as genes that modulate docetaxel chemosensitivity in triple-negative breast cancer. Clin Cancer Res. 2013;19:2061-70.

22. Lavie Y, Fiucci G, Liscovitch M. Up-regulation of caveolae and caveolar constituents in multidrug-resistant cancer cells. J Biol Chem. 1998;273:32380-3.

23. Ando T, Ishiguro H, Kimura M, Mitsui A, Mori Y, Sugito N, et al. The overexpression of caveolin-1 and caveolin-2 correlates with a poor prognosis and tumor progression in esophageal squamous cell carcinoma. Oncol Rep. 2007;18:601-9.

24. Ho CC, Kuo SH, Huang PH, Huang HY, Yang CH, Yang PC. Caveolin-1 expression is significantly associated with drug resistance and poor prognosis in advanced non-small cell lung cancer patients treated with gemcitabine-based chemotherapy. Lung Cancer. 2008;59:105-10.

25. Yang CP, Galbiati F, Volonte D, Horwitz SB, Lisanti MP. Upregulation of caveolin-1 and caveolae organelles in Taxolresistant A549 cells. FEBS Lett. 1998;439:368-72. 